\title{
Estudios
}




\section{GÉNESIS Y HORIZONTES DE ESTUDIO DE LA TEORÍA DE LAS CAPACIDADES}

Diana Ibarra Soto

Centro de Investigación Social Avanzada diana.ibarra@cisav.org

Abstract

The Capabilities Approach has gained preponderance over the past years as a framework for the construction of the political, economical and philosophical life. The following text presents three important aspects of this approach: a) Define and stress the importance of the Capabilities Approach; b) Explain its genesis both historically as well as philosophically; c) Exhibit the scope of options this approach provides to different kind of topics such as human rights, social justice and gender equity. This analysis allows us to redirect the Political Philosophy because it places human being as the center of discussions, in contrast to prevailing contractarian views that had molded most of the twentieth century thought. Nussbaum -influenced by Sen- offers a specific vision that has displaced the disappointment of postmodern deconstructionism toward a more optimistic position closer to the classical philosophy.

Keywords: Capabilities Approach, Martha Nussbaum, Social Justice, Human Rights, Gender Studies. 


\section{1.- Definir y destacar la importancia de La Teoría de las Capacidades}

Martha Nussbaum define las capacidades humanas como: "What people are actually able to do and to be" (Nussbaum, 2000c: p. 5). Desde sus inicios, la teoría de las capacidades ha tenido un objetivo práctico: el mejoramiento de las condiciones mínimas de vida que debe tener una persona para su florecimiento. La teoría de las capacidades, antes que nada, es una propuesta para solucionar las inequidades que presenta el mundo contemporáneo.

Nussbaum entiende por filosofía el servicio que el intelectual hace a la sociedad. Desde sus primeros años de estudio en el doctorado en Harvard, se puede encontrar una constante en sus investigaciones: definir lo "verdaderamente humano". ¿Qué es aquello sin lo cual el humano no es humano? ¿Qué condiciones se necesitan para que esto suceda? ¿Es algo que se encuentra dado o hay que ganárselo? ¿Qué implicación tiene el ser un humano, derivaciones para con uno mismo y para los demás?

De acuerdo con Nussbaum, el filósofo es el principal encargado de esta tarea, crucial para resolver la crisis moral de nuestros tiempos, pues es él quien puede disponer del tiempo para reflexionar en cómo mejorar la situación humana: "Philosophy is not just any old technique of making people happy; it secures the flourishing life by means of reasoning and argument" (Nussbaum, 1992a: p. 1).

De esta forma, la razón humana es la vía idónea de encuentro con un discurso universal. Es evidente que esta teoría tiene detrás la confianza en la posibilidad del acercamiento con la verdad, además de una perspectiva del humano como un fin en sí mismo. "The capabilities are held to be important for each and every person: each person is treated as an end, and none as a mere adjunct or means to the ends of others"(Nussbaum 2003: p. 12). Siguiendo este evidente influjo kantiano, la autora de las capacidades argumenta que el fundamento último para esta valía personal reside en la dignidad:

Human beings have a dignity that deserves respect from laws and social institutions. (...) The idea of human dignity is usually taken to involve an idea of equal worth: rich and poor, 
rural and urban, female and male, all are equally deserving of respect, just in virtue of being human, and this respect should not be abridged on account of a characteristic that is distributed by the whims of fortune. (Nussbaum, 1999: p. 5).

Con este enfoque, Nussbaum opina que hay diez indicadores centrales que todo humano debe poseer para considerar que tiene una vida digna. Si alguna de estas cualidades no se presenta por lo menos en un nivel mínimo, no es posible sostener que hay un verdadero funcionamiento humano (ver Nussbaum, 2006: pp. 70-71).

El listado sobre las capacidades al que se ha hecho referencia es el siguiente:

1) Vida

2) Salud corporal

3) Integridad corporal

4) Sentidos, imaginación y pensamiento

5) Emociones

6) Razón práctica

7) Afiliación

8) Respeto a otras especies

9) Juego

10) Control sobre el propio ambiente

En cada una de estas capacidades, Nussbaum hace aclaraciones pertinentes, y aunque su obra es vasta, cada texto que ha escrito con posterioridad a este enfoque podría situarse en alguna o varias de estas capacidades. Hay dos aspectos que son interesantes de señalar al respecto. Primero, por medio de esta teoría se podría llegar a postular que existe una especie de denominador común para todos los individuos de la raza humana. Este universalismo respondería a las demandas de un consenso general tanto en las organizaciones a nivel local, como en los postulados del derecho internacional. El segundo aspecto va ligado, con esta última idea, y es que cada una de estas capacidades se tiene que desarrollar, pero el modo en que florecen variará de acuerdo con la cultura del individuo, lo que abre la puerta 
al multiculturalismo. Uno de los problemas más actuales y más complejos es el de cómo hacer coincidir las distintas cosmovisiones de los humanos del mundo dentro de relaciones económico sociales cada vez más globalizadas. La flexibilidad del enfoque de las capacidades en parte está pensada para resolver este conflicto latente.

La justicia social para Nussbaum consistirá entonces en velar para que cada integrante de una sociedad pueda, si así lo decide, desarrollar sus capacidades hasta donde le sea posible. "The capability approach considers people one by one, not as parts of an organic unit" (Nussbaum 1999: p. 34). Esta conjunción de respeto a la persona en cuanto tal, con todo su contexto histórico, pensamiento y sentimientos, sumado a la conexión de un lazo unificante en los aspectos más intrínsecos e inmanentes de la existencia, es lo que le da a la oferta nussbaumniana una puerta de entrada a las consideraciones sobre la justicia en el mundo actual.

Y es aquí en donde quisiera destacar la importancia que ha adquirido este planteamiento pues ha sido el parámetro con que actualmente las Naciones Unidas elaboran el Informe sobre el Desarrollo Humano (HDR por sus siglas en inglés), pues hasta el momento ha resultado ser el medio más preciso para identificar las necesidades y oportunidades a nivel mundial. Anteriormente las mediciones económicas utilizaban principalmente el IPC como indicador para la toma de decisiones en cuanto a políticas de ayuda a países con economías deficientes. Sin embargo, este dato poco o nada decía sobre la distribución de la riqueza y los problemas reales que enfrentaba una comunidad. Al tener los diez medidores expuestos anteriormente se ha podido examinar con mayor precisión cuál es la situación objetiva de estas zonas, así como las decisiones más correctas ante un determinado problema.

Si bien la propuesta de Martha Nussbaum lleva detrás un profundo conocimiento antropológico, su prioridad es de orden político: "The idea is that once we identify a group of especially important functions in human life, we are then in a position to ask what social and political institutions are doing about them" (Nussbaum, 1992b: p. 214). La filosofía política mostrada en este enfoque es de orden activo y demandante, no solo de un compromiso interno con el pro- 
pio desarrollo, sino con la elaboración de una conciencia general que vele por las condiciones de la totalidad de los integrantes de una comunidad y que se esmere en corregir los espacios en donde se vea mermada una posibilidad de crecimiento humano, así como de buscar las oportunidades tanto por vía legal, como pública y privada para incrementar las opciones verdaderas que se le brindan a las personas para lograr un perfeccionamiento integral. "Citizens should be given the option, in each area, of functioning in accordance with a given capability or not so functioning. To secure a capability to a citizen it is not enough to create a sphere of non-interference: the public conception must design the material and institutional environment so that it provides the requisite affirmative support for all the relevant capabilities" (Nussbaum, 2003: 31).

Históricamente, es innegable que la teoría de las capacidades es un referente obligado al hablar de justicia social, desarrollo humano, pobreza y equidad en nuestro siglo. Sin embargo, sería falso afirmar que toda esta propuesta le deba su autoría a la mente de una sola persona, es por ello que para dimensionar sus alcances será necesario exponer cuál fue el origen de esta propuesta.

2.- La génesis histórica y filosófica de la teoría de las capacidades

La construcción de la Teoría de las Capacidades ha sido confeccionada con una colaboración múltiple y a lo largo de mucho tiempo. Amartya Sen, premio Nobel de economía, fue el responsable de la construcción de la teoría de la capacidades, en dicha sede.

Sen reúne el conocimiento vertido a lo largo de su infancia en Calcuta y sus más altos estudios en Cambridge. No solo cavila sobre los medios para eliminar la pobreza, sino que quiere descubrir un estándar para evaluar cuáles son las condiciones en las que viven los seres humanos. En 1989 recibe la invitación del economista pakistaní, Magbub al Haq, antiguo compañero del Trinity College en Cambridge, para contribuir al Human Development Report (Reporte de Desarrollo Humano); proyecto solicitado por la ONU para valorar las condiciones de vida de los distintos países del mundo, tanto a nivel 
teórico, como práctico. El reporte inicia con la frase: "People are the real welth of a nation" (HDR, 1990: p. 9). La visión que es coincidente con los escritos senianos es que las posibilidades de crecimiento de una nación no pueden alcanzarse al margen de su gente. Más allá de pensar en materia de producción, armas o industria, Sen plantea que el desarrollo de una nación tiene un referente directo al nivel de vida que tienen cada uno de sus ciudadanos. En el mismo informe se define el desarrollo humano como: "a process of enlarging people's choices" (HDR, 1990: p. 10).

Desde sus primeros años en Calcuta, Amartya Sen respira las inequidades económicas en el mundo. Toda su teoría económica está marcada por una reflexión constante sobre temas como "la elección", "las oportunidades", "la pobreza y la hambruna". Sin embargo, no es sino hasta 1979 que expone por primera vez su teoría de las capacidades frente a la comunidad académica de la Universidad de Stanford con la conferencia titulada "Equality of What?", en donde muestra la importancia de una reformulación en los parámetros de evaluación de las condiciones en las que viven las personas en aras de un equidad mundial.

De esta forma, propone un tipo de equidad que llamará "igualdad de capacidades básicas" (basic capability equality) que consiste en la interpretación de necesidades e intereses en virtud de una demanda por la igualdad. Sen reconoce que la elaboración de un índice de las capacidades básicas es una labor complicada. Pero destaca que cualquier propuesta que pretenda uniformar las preferencias personales debe estar apoyada en el establecimiento de convenciones de una relativa importancia (Sen, 1979: p. 218).

La postura de Sen, en cuanto al enfoque de las capacidades, ha sido complementada por diversos estudiosos. Algunos de ellos se han interesado en elaborar indicadores más concretos y visibles para ser usados como referencias de aplicación de este enfoque. Diversas listas han sido propuestas por la economista y socióloga Sabina Alkire de la Universidad de Oxford (Alkire, 2002); por el filósofo David A. Clark de la Universidad de Manchester (Clark, 2002) por la economista Meghnad Desai - quien fuera directora del Centro de Estudios para la Gobernabilidad Global (Desai, 1994)--; y la filósofa y economista In- 
grid Robeyns de la Universidad de Rótterdam (Robeyns, 2003), entre otros. Las variaciones obedecen en gran parte a los estudios y objetivos de cada uno de estos intelectuales. Pero, sobre todo, por lo que entienden por el humano, la justicia, la igualdad y el gobierno. En palabras de Robeyns: "The capability approach is a framework of thought, a normative tool, but is not a fully specified theory that gives us complete answers to all our normative questions" (Roybens, 2003: p. 64).

Dentro de las propuestas anteriores, la concreción más conocida y con mayor influencia ha sido la de Nussbaum. Para Sen, la adaptación de la teoría de las capacidades tiene que ser por vía democrática dejando que cada comunidad decida lo que es importante: "It is important that people evaluate explicitly and critically what they want, and engage in arguing for-or against-any set of proposed weights. What weights may emerge is ultimately a matter of social choice" (Sen, 2002: p. 21). Sen y Nussbaum tienen una visión distinta del humano, pues mientras ella fija unos indicadores universales, él se va por el voto de la mayoría. Sin embargo, aquí es en donde el papel de Martha Nussbaum se vuelve fundamental pues construirá todo el sustrato filosófico de la propuesta de Sen, proporcionando argumentos de diferente calibre a la teoría original. Dicho por Sen mismo en una entrevista: "Martha Nussbaum has been a mayor contributor to the literature on capabilities. She has made the whole field much more vibrant as well as accessible. She has also created the context in which this field is taken seriously into account not by economist alone, but philosophers as well, and social scientists generally" (Shaikh, 2004: p. 7).

Nussbaum llegó a su elaboración de la teoría de las capacidades a raíz de una invitación en 1987 por parte de Instituto de las Naciones Unidas en Helsinki llamado World Institute for Development Economics Research (UN-WIDER) para colaborar con Sen y proponer una solución a los problemas de inequidad y justicia de las mujeres del Tercer Mundo

A partir de ese momento, Nussbaum ha colaborado de manera activa en la implementación de esta teoría para lograr una mejora a nivel mundial. Muestra de ello no solo son sus libros, conferencias y artículos, sino la creación de la Human Development and Capability Association (HDCA) en 1994, que tiene por objetivo promover los 
estudios tomando como partida el Enfoque de las Capacidades, desde distintas disciplinas, sobre los problemas sociales como la pobreza, la justicia, la equidad, el bienestar y la economía a nivel mundial, aunque muy particularmente en la India.

Pienso que el interés y compromiso que muestra Nussbaum por la Teoría de las Capacidad tiene como principio un cuestionamiento de tipo filosófico muy anterior a su llamado a trabajar para la ONU. Desde sus inicios como joven estudiante de filosofía muestra un interés por el tema del "funcionamiento", muestra de ello es su tesis doctoral Aristotle's De Motu Animalium, en donde haciendo gala de sus vastos conocimientos de filología griega y metodología estricta, dirige prioritariamente el concepto de teleología ha destacar la importancia del "funcionamiento" para la definición de una potencia. Al relacionar esta obra con su producción futura se puede encontrar que Nussbaum en lo que está pensando es en encontrar los actos esenciales, por un lado, de lo propiamente animal y, por otro, de lo concretamente humano. Esta es la razón de ser de The Fragility of Goodnes: Luck and Ethics in Greek Tragedy and Philosophy, su segunda gran obra. Es un texto que trata de reunir lo mejor del pensamiento griego, tanto literario como filosófico, reconociendo las aportaciones que ha hecho para lograr la excelencia humana, sin olvidar mostrar sus errores. El texto empieza de esta manera: "But human excellence grows like a vine tree, fed by the green dew, raised up, among wise men and just, to the liquied sky"(Nussbaum, 1986). La frase es de Píndaro y Nussbaum la encuentra ejemplificadora de los requerimientos de todo ser para encontrarse con su plenitud. Al llevarlo al plano humano, comenta: We need to be born with adequate capacities, to live in a fostering natural and social circumstances, to stay clear of abrupt catastrophe, to develop confirming associations with other human beings" (Nussbaum, 1986).

Me parece significativo encontrar la palabra capacities en este escrito sabiendo que la primera vez que Nussbaum sostiene la teoría de las capacidades como tal es en el libro The Quality of Life, editado junto con Amartya Sen en 1993. Esto revela que el desarrollo de la teoría de las capacidades había comenzado su gestación en la mente nussbaumniana tiempo antes de su contacto con Sen. La propuesta económica del premio Nobel encontró tierra fértil en el pensamien- 
to de una prometedora filósofa americana que sentía un fuerte compromiso con el género humano. De ahí que su principal interés sea de corte práctico, dicho por ella en una entrevista: "For any view you put forward, the next question simply has to be, "What would the world be like if this idea were actually taken up?'” (Boyton, 1993).

A partir de esta directriz, considero oportuno exponer de manera concisa cuáles son algunos de los terrenos de estudio en los cuales la teoría de las capacidades ha incidido y puede incidir de manera contundente.

\section{3.- Exhibir el abanico de opciones de estudio que brinda su acercamiento}

Son muchas las áreas de interés para un estudioso de la teoría de las capacidades. En cualquier dimensión del desarrollo de lo humano, de preocupación por sus manifestaciones y búsqueda de bien común, tiene cabida esta teoría. Trataré de mostrar, en orden temático partiendo de lo político hasta llegar a lo antropológico, algunas cuestiones que considero significativas, en las que se ha demostrado su incidencia.

\section{a) Los derechos}

Al hablar de política en los tiempos actuales es inadmisible no tocar el tema de los derechos. Después de la Segunda Guerra Mundial, la Declaración Universal de Derechos Humanos es un lugar común al exponer lo que es justamente dado a los humanos. Sin embargo, en general, al momento de llevar la concepción de los derechos al plano material se ensucia con los intereses de los grupos dominantes.

"La república (...) se constituye como comunidad de derechos que iguala a sus integrantes por el estatuto jurídico de la ciudadanía” (Rosales, 1999: p. 139). Los derechos son cartas de reconocimiento dadas por una autoridad a las personas. Ante todo, son normas políticas, resultado de discusiones. El Estado es quien los garantiza. 
El problema se presenta cuando hay que definir quiénes son los que integran el Estado, quiénes los que son reconocidos como autoridad y cuáles son los acuerdos, "derechos", que estipulan. El "derecho", entonces, sería una construcción humana, dada por un consenso, por lo que al no haber consenso o estar dividido no podríamos pedir los mismos derechos para todos.

Aún más, como es sabido, no son pocas las críticas en torno a la concepción eurocentrista de la Declaración de los Derechos Humanos. Nussbaum intentará ofrecer respuesta mediante La Teoría de las Capacidades pues complementa la visión de los derechos. "Thus capabilities have a very close relationship to human rights, as understood in contemporary international discussions. In effect they cover the terrain covered by both the so-called "first-generation rights" (political and civil liberties) and the so-called second generation rights (economic and social rights). And they play a similar role, providing both a basis for cross-cultural comparison and the philosophical underpinning for basic constitutional principles" (Nussbaum, 2000b: p. 2).

Nussbaum aclara que la idea de las capacidades frente a los derechos difiere en tres aspectos:

i) En cuanto a la claridad de lo que implican los derechos pues hay quien entiende por ellos algo puramente consensual, mientras que otros hacen referencia a un ius naturalismo; unos entienden por derechos algo que involucra solamente a los individuos mientras otros hablan de derechos colectivos. También difieren en la relación que hay entre derechos y obligaciones y si todos los derechos tienen implícita una obligación.Y por último si los derechos deben ser vistos como restricciones de acciones en cuanto al límite de la existencia de los otros, o como objetivos de bienestar que hay que tener en mente (Nussbaum, 2000b: p. 11).

ii) Las capacidades se enfocan en el funcionamiento y no solamente en la promulgación de una ley. El problema es que la ley muchas veces no toma en cuenta los recursos que en efecto se tienen para vivir ese derecho. 
iii) El enfoque de las capacidades tiene en la mira el respeto de las diferentes ideologías culturales: "When we speak simply of what people are actually able to do and to be, we do not even give the appearance of privileging a Western idea. Ideas of activity and ability are everywhere, and there is no culture in which people do not ask themselves what they are able to do, what opportunities they have for functioning" (Nussbaum, 2000: p. 12).

Se podría plantear que el lenguaje de las capacidades privilegia la elección personal frente a un cúmulo de oportunidades reales, presentes en el mundo y accesibles a la persona, mientras que los derechos crean las posibilidades jurídicas para asegurar e incentivar las acciones normativas en un determinado Estado. Sería un error pretender que un planteamiento supera o sustituye a otro. Ambos tienen dimensiones y pretensiones distintas y pueden favorecerse mutuamente, tanto en su plano teórico como en sus construcciones prácticas.

\section{b) La justicia social}

La preocupación por lograr la justicia tiene como objetivo la convivencia pacífica entre los hombres. Es evidente que ante la diversidad de intereses de los integrantes de una población es frecuente que surjan fricciones ante la incompatibilidad de finalidades. De manera directa la teoría de Nussbaum está encaminada a la distribución de recursos y oportunidades teniendo como marco de referencia las capacidades: "a particular list of capabilities ought to be used to define a minimum level of social justice, and ought to be recognized and given something like constitutional protection in all nations" (Nussbaum, 2005: 175).

Siguiendo las enseñanzas del actual director de la Escuela de Frankfurt, Axel Honneth, la justicia no solo trata de distribuir los bienes sino, primero que nada, de reconocer que a lo que se está observando es otra persona, una que responde en igualdad de condiciones a todo aquel que tenga la misma esencia antropológica. Este 
"reconocimiento" de la humanidad es lo que permitirá establecer relaciones de tipo horizontal, de paralelismo entre las personas, independientemente de la cultura, sexo o clase social a la que se pertenezca. Honneth está consciente que el asesinato, el robo, la humillación, la mentira, toda injusticia social tendrá detrás una falta de reconocimiento (Honneth 2004: p. 352). "El problema se agrava cuando se sabe que la autoconciencia del ser humano depende de la experiencia del reconocimiento social” (Honneth, 1998: p. 23). La percepción que se tiene de sí mismo en mucho depende de la mirada con la que se es tocado. Las relaciones que se sostienen con las personas circundantes marcan una huella en cada uno, que hace que uno se identifique con determinada cualidad, obligación o derecho. "En el trato social se establece la conducta del día a día. Las palabras con las que se designan a los humanos que tienen un trato contiguo a nuestra existencia imprimen un sello en nuestra identidad. Así, el crimen social, el menoscabo a la dignidad del otro, imprime una herida moral, por la que las personas son lesionadas en el entendimiento positivo de sí mismas" (Honneth, 1997: p. 160).

Es por eso que el reconocimiento de las capacidades permitirá por un lado un fortalecimiento en la cohesión social y, por otro, la creación de identidades que puedan asumir un papel activo en su comunidad: que los individuos al pensarse se reconozcan con posibilidades diversas y dinámicas, fruto de sociedades justas en donde se les exija que colaboren con el establecimiento y preservación de la justicia. "The view developed here seeks justice for human beings as such, believing all human beings to be fundamentally equal in worth" (Nussbaum, 1999: p. 7).

c) Los reduccionismos identitarios de género

Una veta de estudios en la que la teoría de las capacidades tiene una participación continua e innegable es la de los estudios de género. No se puede incursionar en estas investigaciones si no se tiene claro que el sexo no es el género, pero que la relación entre ambos es confusa. La sociedad asume una serie de juicios ciertos de un individuo tan 
solo por el hecho de tener un sexo definido y conformará su género en parte tomando en cuenta la voluntad del interesado, pero en su mayoría conformándolo sin que él mismo se percate. No hay identidad humana que no esté atravesada por el género pero ¿hasta dónde llega el sexo y hasta dónde el género? Estamos frente a una pregunta que no está resuelta.

Con la intención de resumir este problema, de una manera expositiva me inclino a condensar el espectro de opiniones a dos principales: los estudios sociobiologisistas y la interpretación performativa.

En los estudios de género, la sociobiología ha cobrado importancia al tratar de determinar los influjos biológicos que desencadenan un determinado comportamiento sexual en la sociedad. El problema se presenta cuando se hace una conexión necesaria entre una estructura anatómica o fisiológica con un desempeño cultural; como sería el suponer que dado el diformismo sexual cerebral, las mujeres tienen siempre un desempeño más pobre que el varón en las matemáticas o en la rotación de objetos mentales, mientras que muestran una mayor expresión verbal o una mejor percepción de los detalles (Kimura, 2003). Si bien los resultados suelen matizarse de acuerdo a la edad y a la educación, es común caer en determinismos al visualizar al humano solamente con un cúmulo celular evolucionado. En el ámbito feminista de los estudios de género esta postura es ampliamente criticada pues abre la puerta a las estructuras de dominación existentes. "Feminist sociobiology does not represent progress for feminist evolutionary science because it suggests a biological essentialism at the heart of human behaviour" (Sperling, 1991: p. 250). Lo que se suele llama "esencialismo" se interpreta como algo dado e inamovible, por lo que la evolución humana desde una perspectiva sociobiologisista supondría que el hombre se ha desarrollado de una manera superior a la mujer y que eso ha traído consigo la definición de los roles genéricos existentes. "La determinación fisiológica implica mucho más que el hecho de que sean las mujeres, y no los hombres, quienes gestan y alimenten a los hijos. El término conlleva la imagen de una forma femenina intrínsecamente inferior, en particular de una forma de inteligencia inferior, que relega naturalmente a todas las mujeres a la ejecución de ciertas tareas bien delimitadas" (Williams, 1973: p. 1,725). 
Por otro lado, la performatividad sugiere una presencia de lo humano antiteleológica. Prototípicamente, la derridariana Judith Butler encabeza este movimiento que niega cualquier tipo de concepto metafísico o determinante y opta por la creación libre y continua que adquiere y pierde su significado al momento de ser realizada. "Si el género es los significados culturales que asume el cuerpo sexuado, entonces no puede decirse que un género sea resultado de un sexo de manera única. Llevado hasta su límite lógico, la distinción sexo/ género indica una discontinuidad radical entre cuerpos sexuados y géneros culturalmente construidos" (Butler, 2007: p. 39). No hay razón para ligar el sexo con el género. De hecho, no hay razón para voltear a ver al sexo (Cucchiari, 1996: p. 210). Todo en el humano sería una creación social, ya sea conciente o inconsciente, pero no habría nada de natural que impusiera su curso. Toda la tradición histórica podría perderse de un plumazo y junto con ella las estructuras de dominación masculina imperantes. Si esto es verdad, la pregunta que habría que hacer entonces es: ¿cómo determinar la conveniencia de la construcción personal? ¿Cómo formar una conciencia, por no apelar a la rectitud, de lo que me proporcionará un mayor progreso? Dentro de los muchos problemas que presenta la propuesta performativa está el que le pide al humano dejar de pensar en el futuro y guiarse por la razón. Pese a que esta es una filosofía de inconsistencia, la cantidad de seguidores que posee es considerable.

Encontrar un punto medio entre lo natural, incluyendo lo biológico, y lo socialmente construido sería un modo de resolver las diferencias entre estas dos posturas y promover una modificación de estructuras sociales sin perder la esencia del humano. Pienso que la teoría de las capacidades es idónea para resolver este conflicto al centrarse en características universales que poseen todos los humanos en cuanto tales, pero que se desenvuelven en virtud de los influjos que reciben del medio. "Questions of life and death, of good and bad nutritional status, of fitness and strength, of good and ill health, are not simple maters of cultural advertising, thought, of course, experiences that are culturally shaped may influence them in many ways" (Nussbaum, 1998: p. 29). El humano es una realidad que tiene una conformación heterogénea, pero que requiere unas constantes para 
poder desarrollarse de una manera idónea. Si no detectamos cuáles son estas constantes y qué es lo propiamente humano, no se podrá velar por su incremento; al mismo tiempo, dicho incremento deberá estar mediado por una sociedad, la cual es en cierta medida responsable de los estímulos que recibe la persona y de las asociaciones que vincula a determinados comportamientos que juzga "normales". Esta distinción entre lo dado y lo creado en la naturaleza nos podrá proporcionar una directriz mayor al momento de tomar no solo decisiones individuales sino políticas en virtud de un esencialismo dinámico. "I argue that without essentialism of a kind, we are deprived of two moral sentiments that are absolutely necessary if we are to live together decently in the world: compassion and respect" (Nussbaum, 1992a: p. 205).

\section{d) Equidad de género}

"Many women all over the World find themselves treated unequally with respect to employment, bodily safety and integrity, basic nutrition and health care, education, and political voice" (Nussbaum, 1999: p. 5). Una ideología masculina dominante ha provocado un juego de construcciones en donde la mujer ha salido perdiendo, identificada muchas veces con "cabellos largos e ideas cortas". La discriminación de la mitad de la raza humana se puede encontrar aún en nuestros días en muchas áreas del desenvolvimiento personal. La búsqueda por la igualdad, no solo a nivel teórico sino práctico, muestra la importancia de definir una de las principales demandas a la sociedad actual: lograr un desarrollo óptimo de todos sus ciudadanos con independencia del sexo. "In order to highlight these inequities

and provide a rationale for their readdress, I have been developing the "capabilities approach" to understanding women's human development. This approach measures the presence of basic opportunities by looking at what individual women are actually able to do and to be” (Nussbaum, 2000a: p. 175).

Muchas de las actividades propiamente ligadas con la feminidad han sido utilizadas como medio de supresión de la mujer; no porque 
estas actividades, de suyo, obedezcan a una inferioridad de condiciones sino porque la interpretación social de este quehacer femenino las ubica dentro del rubro de actividad de poca importancia, como la crianza de los hijos o cuidado del hogar. Dicha valoración establece una relación de dominio de unos hombres sobre otros. Al buscar una sociedad en la cual la supresión del otro es fundamental para lograr la afirmación del yo, se menosprecia la dinámica de una vida íntima o afectiva pues se entiende como inoperante. Las actividades femeninas no se han identificadas como fundadoras de una autoridad sobre las demás sino al contrario, como medios para el sostenimiento del poder masculino. Además, los matrimonios arreglados, el gineceo, la dote son figuras sociales anexadas al ser femenino que han colaborado a que se identifique dicha naturaleza con el sometimiento.

La teoría de las capacidades surge como una propuesta de solución para focalizar las funciones centrales de los humanos, postulando un universalismo que ayude a soslayar los problemas de las mujeres, particularmente en el mundo en desarrollo. Se busca instaurar unos indicadores que sirvan para medir la situación en la que están ubicadas las mujeres de acuerdo a su entorno: si tienen derecho a la educación, a la propiedad, a trabajar o a participar en al vida política. La teoría de las capacidades está diseñada para dar soluciones reales a problemas reales. Esta es la propuesta filosófica de Nussbaum: "The capabilities approach is indeed a valuable way to approach the question of fundamental entitlements, one that is especially pertinent to issues of sex equality" (Nussbaum, 2000a: p. 173).

Nussbaum sostiene que la teoría de las capacidades no está acabada y que debe estar bajo el escrutinio constante de cada sociedad en la que se aplique. "I have leave things deliberately somewhat openended at this point, in accordance with the procedures of the Human Development Report, believing that the best way to work toward a more precise determination, at present, is to focus on comparative information and to allow citizens to judge for themselves whether their policy makers have done as well as they should have" (Nussbaum, 1999: pp. 42-43). De esta forma, la autora deja abierta la solución de varios asuntos, pues confía que mediante la participación social específica de cada uno de los grupos aludidos, se logre una acercamiento 
respetuoso entre los ideales propuestos y las costumbres propias de cada una de las sociedades y de sus miembros.

El proyecto nussbaumniano tiene una clara orientación práctica que se revela no solo en sus elucubraciones de filosofía política sino, sobre todo, en el que plantea su vida, pues para Nussbaum la teoría conlleva necesariamente un compromiso de acción: "Most of all, I think, I want to be, and I want my students to see me to be, a person passionately immersed in the ethical issues of life, one who does not shrink from taking risks for those commitments, but also one who loves philosophy and believes that the best way to address these commitments is through the very sort of reasoning and study that I am asking them to do, which I see as something extremely joyous and deeply delightful" (Nussbaum, 1992b: p. 3). 


\section{BiBLIOGRAFÍA}

A.A.V.V., 1993. The Quality of Life, Nussbaum, Martha y Sen, Amartya, eds., Oxford: Clarendon Press.

A.A.V.V., 1990. Human Development Report, 1990, Ul Haq, Mahbub, director del proyecto, New York: United Nation Development Program \& Oxford University Press.

Alkire, Sabina, 2000. Valuing Freedoms: Sens's Capability Approach and Poverty Reduction, Oxford: Oxford University Press.

Boynton, Robert, noviembre de 1999. "Who Needs Philosophy? A profile of Martha Nussbaum” en New York Times Magazine, 21 (99).

Butler, Judith, 2007. El género en disputa, Paidos, Col. Género y sociedad.

Clark, David A., 2002. Visions of Development: A study of Human Values. Cheltenham: Edward Elgar.

Cuchiari, Salvatore, 1996. "La jerarquía de género: Teorías de los orígenes versus teorías de la reproducción” en El género: la construcción cultural de la diferencia sexual, México: PUEG-UNAM.

DesaI, Meghnad, 1994. "Poverty and Capability:Towards an empirically Implementable Measure” en Poverty, Famine, and Economic Development, Aldershot: Edward Elgar.

Honneth, Axel, 1997. La lucha por el reconocimiento, Barcelona: Crítica Grijalbo Mondadori.

Honneth, Axel, 1998. "Entre Aristóteles y Kant. Esbozo de una moral del reconocimiento” en Lógos, Anales del Seminario de Metafísica, Madrid: Servicio de Publicaciones, Universidad Complutense.

Honneth, Axel, 2004. "Recognition and Justice: Outline of a Plural Theory of Justice" en Acta Sociológica, 47.

Kimura, Doreen, 2003. “Sex Differences in the Brain” en Science American, mayo 13.

Nussbaum, Martha, 1986. The Fragility of Goodness: Luck and Ethics in Greek Tragedy and Philosophy, Cambridge: Cambridge University Press.

Nussbaum, Martha, 1992a. "Human Functioning and Social Justice, in Defence of Aristotelian Essentialism” en Political Theory, vol. 20 no. 2, may.

Nussbaum, Martha, 1992b. "No Chance Matter. Philosophy and Public Life" en Teaching the Classics, Brown Classical Journal, vol. 8, Supplement.

Nussbaum, Martha, 1998. "Constructing Love, Desire, and Care" en Sex, Preference and Family: Essays on Law and Nature, Estund, David M., ed., NC, USA: Oxford University Press.

Nussbaum, Martha, 1999. Sex and Social Justice, New York: Oxford University Press. 
Nussbaum, Martha, 2000a. The “Capabilities”Advantage to PromotingWomen's Human Rights, agosto 6.

Nussbaum, Martha, 2000b. "Women and Work-the capabilities approach" en The little magazine, vol. I, issue 1.

Nussbaum, Martha, 2000c. Woman and Human Development, Cambridge: Cambridge University Press.

Nussbaum, Martha, 2003. "Capabilities as Fundamental Entitlements: Sen and Social Justice”, Feminist Economics, vol. 9, no. 2 \& 3 (jul./nov.): pp. 33-59.

Nussbaum, Martha, 2005. “Women’s Bodies:Violence, Security, Capabilities” en Journal of Human Development, 6(2): pp. 167-183.

Nussbaum, Martha, 2006. Frontiers of Justice. Disability, Nationality, Species Membership, Londres: Belknap Press.

Robeyns, Ingrid, 2003. "Sen's Capability Approach and Gener Inequality: Selecting Relevant Capabilities” en Feminist Economics, 9(2-3): pp. 61-92.

Rosales, José María, 1999. "Patriotismo constitucional: sobre el significado de la lealtad política republicana” en Isegoría: Revista de filosofía moral y política, no. 20: pp. 139-149.

SEn, Amartya, 1979. Equality of What? The Tannner Lecture on Human Values, Stanford: Stanford University, may. 22.

Sen, Amartya, 2000. "A Decade of Human Development" en Journal of Human Development, vol. 1, no. 1, Carfax Publishing.

SHAikh, Nermeen, 2004. "Development as Freedom: Asia Source Interview with Amartya Sen” en Asia Source, dic. 6.

Sperling, Susan, 1991. "Baboons with Briefcases vs. Langurs in Lipstick" en Gender at the Crossroads of Knowledge Di LeOARDo, M., ed., Berkeley: Uniersity of California: pp.204-234.

Williams, Neely, 1973. "The Argument Against the Physiological Determination of Female Roles: a Reply to Pierre L.Van den Berghe's Rejoinder to Williams Extensions of Brown's Article" en Amercian Anthopologist, 75: pp. 1,725-1,728. 
